\title{
A Negotiation Game for Multichannel Access in Cognitive Radio Networks (Invited Paper) *
}

\author{
Hua Liu \\ Department of Computer \\ Science \\ University of Southern \\ California \\ Los Angeles, CA 90089 \\ hual@usc.edu
}

\author{
Longbo Huang \\ Ming Hsieh Department of \\ Electrical Engineering \\ University of Southern \\ California \\ Los Angeles, CA 90089 \\ longbohu@usc.edu \\ Qing Zhao \\ Department of Electrical and \\ Computer Engineering \\ University of California, Davis \\ CA 95616 \\ qzhao@ece.ucdavis.edu
}

\author{
Bhaskar Krishnamachari \\ Ming Hsieh Department of \\ Electrical Engineering \\ University of Southern \\ California \\ Los Angeles, CA 90089 \\ bkrishna@usc.edu
}

\begin{abstract}
We consider the problem of efficient opportunistic spectrum access in cognitive radio networks where there are multiple secondary users trying to share access to multiple channels. In our formulation, each user has a potentially different valuation of each channel and wishes to pick a channel in such a way as to maximize its benefit without interfering with other users. There is a fundamental tradeoff in this problem - while information about other secondary users is useful in making a good channel sensing/access decision, the communication cost of gathering this information must be taken into account. We formulate the problem as a multi-round negotiation game in which the users try to gather "just-enoughinformation" to make their decisions. The channel valuations are modeled as independently uniformly distributed random variables between 0 and 1 . We propose a thresholdbased channel sensing policy based on observations from a previous work. For a two-user two-channel setting, we calculate optimal thresholds, and obtain the corresponding performance for cases with no information exchange, partial information exchange, and full information exchange. We then show how the optimal amount of information exchange varies with the cost of negotiation.
\end{abstract}

\section{Categories and Subject Descriptors}

C.2.5 [Computer Systems Organization]: COMPUTER-

\footnotetext{
${ }^{*}$ This work was supported in part by the National Science Foundation under Grant CNS-0347621 and by the Army Research Office under Grant W911NF-08-1-0467.
}

Permission to make digital or hard copies of all or part of this work for personal or classroom use is granted without fee provided that copies are not made or distributed for profit or commercial advantage and that copies bear this notice and the full citation on the first page. To copy otherwise, to republish, to post on servers or to redistribute to lists, requires prior specific permission and/or a fee.

WICON'08 November 17-19, 2008, Maui, Hawaii, USA.

Copyright 2008 ICST 978-963-9799-36-3.
COMMUNICATION NETWORKSLocal and Wide-Area Networks[Access schemes]

\section{General Terms}

Algorithms, Theory, Design, Performance

\section{Keywords}

cognitive radio, opportunistic spectrum access, multiple secondary users, channel negotiation

\section{INTRODUCTION}

Opportunistic spectrum access (OSA) with cognitive radios has been receiving increased attention from the research community due to its promise for enabling the efficient use of limited spectrum resources [1]. Opportunistic sharing takes advantage of unused spectrum in licensed bands to improve the spectrum utilization. Spectrum that is licensed to a set of primary users may not be used in a particular area or for particular periods of time by these users. Secondary users equipped with cognitive radios are able to detect such holes in the spectrum and communicate without interfering with the primary users.

Channel sensing policy for tracking the white space in the spectrum is an important component of OSA technology. Most existing work has studied the case of a single secondary user $[2,4,8,9,10]$. However, when there exist multiple secondary users within interfering range of each other contending for opportunities, collaboration among secondary users to avoid collisions becomes a key issue that affects the efficiency of spectrum utilization.

We investigate how secondary users should coordinate with each other on channel sensing and access in cognitive networks. We consider a negotiation process where secondary users exchange information about their own valuation of channel conditions with other interfering neighbors in each round. In particular, we consider the scenario where the users' channel valuations, representing the probability that 
they will sense a primary user on that channel, are uniformly randomly distributed between 0 and 1 .

In a recent [14], we considered the same problem with two-users, two-channels, under the assumption that channel valuations are two-valued. An important observation we made in that work is that in case of limited information about the other user, randomizing on the choice of sensing channel will not help improve the expected utility unless the two channels are equally good to the user. We showed that when the channel valuation is limited to be one of two values ("high" or "low"), when there is ambiguity about the valuations of the other user, the secondary user's optimal choice on picking a channel to sense is to either choose the "high" channel with probability 1 (when the user has different valuations on the two channels) or sense each channel with probability $\frac{1}{2}$ (if the user has the same valuation on both channels). We adopt a similar threshold policy in this work, where we extend the previous work to a scenario with uniformly distributed valuations.

For a two-user two-channel scenario, we calculate the user's expected throughput in a given time period for a) conducting no negotiation, b) one round of negotiation and c) two rounds of negotiation. In a 2-user 2-channel case, these correspond to having a) no information about the other user's valuations, b) having partial information regarding the other user and c) having perfect information regarding the valuations, respectively. These calculations use the threshold sensing policy for the cases with no negotiation and oneround of negotiation. For these cases, we obtain the threshold value that maximizes the user's expected throughput.

Using our analysis of the three scenarios, we address the question of the optimal level of information exchange. Exchanging information can reduce the probability of collision among secondary users, but at the same time the exchange occupies valuable time which could be used for channel access. We show that the optimal number of negotiation rounds changes with the ratio of each round's length to the given time interval (which quantifies the cost of negotiation).

The paper is organized as follows. Section 2 discusses related works in the literature. Section 3 describes the background knowledge, including a brief introduction on the negotiation process, the definition of utility function and the previous research conclusion which motivates the threshold policy used in this work. In section 4, we present the threshold and optimal decision calculation for the case where the users' channel valuations are uniformly randomly distributed between 0 and 1 for each of the cases. We also illustrate the analysis with a plot to discuss the user's optimal negotiation rounds as a function of the negotiation cost. Finally, we conclude the paper and list possible future works in section 5 .

\section{RELATED WORKS}

Researchers have been applying game theory broadly to medium access and spectrum sharing in wireless networks for years in non-OSA settings. For example, Mackenzie and Wicker formulate and analyze slotted Aloha medium access with multiple users as a non-cooperative game in [15]. Halldorsson et al. [16] study a channel assignment game in $\mathrm{WiFi}$ networks. Konorski [17] analyzes CSMA/CA protocols from a game theory perspective of view. Cao and Zheng [3] consider the case of sharing common spectrum resource in a multi-terminal wireless ad-hoc network and propose a bar- gaining mechanism to maximize system fairness.

In the cognitive radio context, previous research has mostly focused on single user sensing policies. Zhao et al. [8, 9] show that when modeling the channel sensing decision as a partially observed markov decision process (POMDP) [18], the myopic policy has a simple structure and is provably optimal for the single user in the case of two independent identically evolving Markovian channels. Further results by Javidi et al. [10] and Ahmad et al. [11] have considerably generalized the conditions under which a myopic scheme is optimal. Chang and Liu [4] consider a setting where a single secondary user has to probe multiple channels before making accessing decision. They also consider the tradeoff between the time spent in sensing decision and using the channel, which is similar in spirit to the negotiation/use tradeoff that we consider in this work.

Collision among secondary users becomes a key issue affecting spectrum utilization when there exist multiple secondary users. However, comparing to the studies in other areas, the literature on multiple-channel multiple-user opportunistic spectrum access is limited. Liu et al. [7] provide simulation results to compare the system throughput for four different sensing policies in 2-user 2-channel case when the channel status is modeled as a POMDP [2]. In this case, the distributed myopic policy (i.e. users make myopic decision without considering the existence of the other user) performs worse than the policies with user cooperation in most cases. Similar results are observed in a work by Liu et al. [12] where a distributed randomized policy is proposed within the POMDP framework to address the tradeoff between choosing the channel that is most promising to be idle and avoiding other competing secondary users. These results convince us that coordination is needed in the multiuser scenario.

Fu and van der Schaar [5] have recently modeled the spectrum sharing problem in cognitive radio networks as repeating auctions with a centralized spectrum manager. The secondary users learn from previous auction allocation results and adjust their bidding policy dynamically to maximize their reward. Unlike their work, the objective of this work is to optimize coordination process among secondary users to maximize the user's expected throughput in a one shot game.

Liu and Krishnamachari [6] give a solution to the static case sensing decision with both symmetric and asymmetric cases. One of their conclusions is that in the asymmetric case (i.e., secondary users have independent valuations on the same channel), the optimal system throughput can be reached by applying Hungarian algorithm [13] if all the channel valuations are known to every user in the system. This conclusion is used in this paper. In this paper, instead of considering the case with perfect system information, we discuss the tradeoff between information gathering and channel use.

In Liu et al. [14] we recently discussed a similar problem as this paper where the channel valuations are limited to only two discrete values, "high" and "low". In this work, based on a key observation of the 2-value case, we consider a more general case where the channel valuations for both users are uniformly randomly distributed between 0 and 1 .

\section{PRELIMINARIES}

In this section, we present preliminary background infor- 


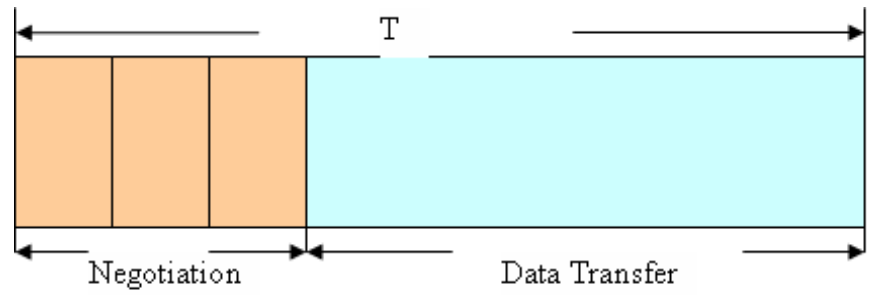

Figure 1: An illustration of the frame containing negotiation rounds

mation. We describe the negotiation process among the secondary users, as well as the decision making process for sensing and access. We also describe the utility function formally.

\subsection{The Negotiation Process}

We consider a given time period of length $T$. There are multiple secondary users in interference range of each other that wish to choose a channel to sense for this time interval. Each user has an independent valuation for each channel that is assumed to be distributed as a uniform random variable between 0 and 1 . The valuation can be taken to indicate the estimated probability that the channel will be free of a primary user for the entire duration. Secondary users may exchange information on their channel valuations with other users to help avoid collisions.

The given time interval $T$ contains two stages: the negotiation stage and the sensing/transmission stage. The negotiation stage may contain several rounds and each round occupies $t$ time units. In negotiation round $k$, every user in the negotiation process shares his/her $k^{t h}$ best channel's valuation and the corresponding channel identity with all his/her neighbors. If there is more than one channel valued the same to a user, the user will uniformly randomly pick one among these channels to share the information. Figure 1 illustrates a typical frame containing 3 negotiation rounds.

The number of negotiation rounds to be followed is assumed to be fixed a priori and known to all secondary users at the beginning of the given time period. We assume that an extra low-rate communication channel exists for the purpose of information exchange between secondary users.

\subsection{The Sensing and Access Process}

Each secondary user decides on a channel and senses the corresponding channel according to the information obtained in the negotiation process. We assume that the channel sensing time is negligible comparing to the negotiation and channel access process. If the channel is sensed to be free of the primary user, the secondary user will commence transmission on this channel for the rest of the time period. A collision happens if more than one secondary user transmits data on the same channel. A transmission is successful if there is no collision. We assume that the throughput for a secondary user in a collision is zero.

The sensing decision is made as follows. If the optimal sensing decision can be obtained deterministically without ambiguity from the gathered information (e.g., there are no conflicts among users' most preferred channels, or the opti- mal sensing/access channel allocation can be derived using a deterministic algorithm), we suppose the users can intelligently use the optimal sensing decision. Otherwise, in case of ambiguity, the secondary users will use a threshold-based policy that we describe in section 4.1.

Specifically, in the two-user two-channel case, the thresholdbased sensing policy is used for two cases: 1) no negotiation and 2) after one-round negotiation if the two users have the same best channel. The remaining cases are handled as follows. In the case of two-round negotiation, each user has global information on each other's valuations for both channels and can determine the optimal sensing decision. In the case of one-round negotiation, if it turns out that each user prefers a different channel, the optimal sensing policy is simply that each user picks their preferred channel.

\subsection{The Utility Function}

The secondary users aim to maximize their own expected throughput in a given time interval $T$. Mathematically, the utility function for user $i$ can be presented as following:

$$
U_{i}=(T-a t) \mathbb{E}(R)
$$

where $a$ is the number of negotiation rounds and $\mathbb{E}(R)$ is the expected throughput in unit time ( $R$ denotes the throughput rate).

In this paper, we consider a one-shot game in the given time slot (i.e., $T$ is a given constant). Instead of having this constant $T$ in the utility function, we model the negotiation cost as the ratio of time for each round negotiation to the total time length $T$. Specifically, we consider an equivalent formulation $\widehat{U_{i}}=\frac{U_{i}}{T}$ as follows:

$$
\widehat{U_{i}}=(1-a \beta) \mathbb{E}(R)
$$

where $\beta=\frac{t}{T}$. Without loss of generality, we assume that each user gets unit data rate on each channel if there is not primary interference or collision with other secondary users ${ }^{1}$ With this assumption, $\mathbb{E}(R)$ becomes a function of the sensing policy and the channel valuations.

In the discussion below, we will use this assumption and the unit time utility function in equation (2) for simplicity.

\section{THE 2-USER, 2-CHANNEL CASE}

In this section, we discuss the specific scenario that involves two secondary users $(P 1$ and $P 2)$ and two communication channels $(C 1$ and $C 2)$. We consider the case that each secondary user's channel valuations are random variables that are independently uniformly distributed between 0 and 1. For a particular channel, each secondary user's valuations are also independent.

By using the insights we get from our previous work, we propose a threshold-based sensing policy for the secondary users. The threshold is common for both users and it is predefined before the users obtain their own channel valuations. The threshold is decided such that the expected throughput rate for the user is maximized. Once the threshold is given, the users need to decide how many rounds of negotiation they should pursue in order to maximize their expected utility (i.e., expected throughput in given time period $T$ ). We

\footnotetext{
${ }^{1}$ Though we omit the details, the users can adjust their channel valuations to consider the case where the data transmit rate is different for them on different channels.
} 
will present a plot based on the analysis to show how the optimal number of rounds changes with different negotiating costs.

We reiterate that the number of rounds of negotiation to be used, and the threshold value to be used, are decided $a$ priori before the particular channel valuations are observed. The analysis for the optimal threshold values and for the number of optimal rounds of negotiation that we provide in this paper are therefore based on averaging over all the possible channel valuations.

Note also that although we only calculate the optimal threshold values by maximizing user $P 1$ 's throughput rate, due to symmetry, this also maximizes the sum-rate of the two users.

\subsection{Threshold-based Sensing Policy}

We propose a threshold-based policy to be used when the information gathered from the negotiation stage leaves some ambiguity about the optimal channel sensing decision. The threshold policy is essentially as follows; if the two channels are "almost equally good" to the user (i.e., difference of channel valuations is less than threshold $\theta$ ), the user will randomly pick one of the two channels to sense with equally probabilities ( 0.5 for each channel). Otherwise, the user chooses the channel with larger valuation.

As mentioned before, this policy is motivated by observations from our previous work [14], where we have considered a similar 2-user 2-channel problem where the secondary users' channel valuations are limited to be one of two values: "high" or "low". In the 2 -value case, in case of ambiguity about the other user's channel valuations, we showed that randomization on channel sensing can help, but only when there is a tie (i.e., when both channels are "equally good" for a user). Otherwise, the user will just pick the better channel according to his/her own information.

The notations and formal description of the sensing policy are given below.

Let function $g_{i}\left(p_{i, 1}, p_{i, 2}\right)$ denote the probability that user $i$ senses channel $C 1$ (i.e., user $i$ will sense channel $C 2$ with probability $\left.1-g_{i}\left(p_{i, 1}, p_{i, 2}\right)\right)$. As mentioned before, we assume that the optimal sensing decision for user $i$ is related to the difference of two channels' valuations based on the observations in 2-value case. That is, the optimal sensing decision $g_{i}^{*}($.$) is a function of p_{i, 1}-p_{i, 2}$. Mathematically, the optimal policy on deciding the probability to sense channel $C 1$ is presented in equation (3).

$g_{i}^{*}\left(p_{i, 1}, p_{i, 2}\right)=g_{i}^{*}\left(p_{i, 1}-p_{i, 2}\right)=\left\{\begin{aligned} 0.5 & \left(\left|p_{i, 1}-p_{i, 2}\right| \leq \theta\right) \\ 1 & \left(p_{i, 1}-p_{i, 2}>\theta\right) \\ 0 & \left(p_{i, 2}-p_{i, 1}>\theta\right)\end{aligned}\right.$

where $\theta$ is a positive number in the interval $[0,1]$.

As we have mentioned, this threshold-based sensing policy is applied only in the cases of no-rounds of negotiation and one-round of negotiation when the optimal channel allocation cannot be determined trivially by the information obtained. For example, after one-round negotiation, if user $P 1$ prefers channel $C 1$ and user $P 2$ prefers the other channel, the optimal channel sensing policy is for the two users to pick their respective preferred channels and the threshold policy need not be applied.

\subsection{Analysis of Optimal Thresholds and Cor- responding Expected Utility}

The users decide the number of rounds to participate in the negotiation a priori in order to maximize their expected utility in the given time period. Specifically, in the twochannel scenario, there are three cases to be considered: 1) user does not participate in the negotiation at all; 2) user takes one round of negotiation before deciding on the channel to sense and access; 3) user gains full information on the other through two rounds of negotiations. In the first two cases, the value of $\theta$ might effect the user's expected utility in unit time. We obtain the optimal $\theta$ that maximizes user's expected utility in unit time for these cases.

Without loss of generality, we consider secondary user $P 1$ in the following. We also assume without loss of generality that for the considered user, channel $C 1$ is better than channel $C 2$ (i.e., $p_{1,1} \geq p_{1,2}$ ).

\subsubsection{No Information Exchange}

Consider first that the user does not participate in the negotiation and no information is exchanged. The expected throughput rate for user $P 1$ is given by expression (4).

$$
\begin{aligned}
& \int_{p_{1,1}} \int_{p_{1,2}} \int_{p_{2,1}} \int_{p_{2,2}} {\left[g_{1}^{*}\left(p_{1,1}, p_{1,2}\right) p_{1,1}\left(1-g_{2}^{*}\left(p_{2,1}, p_{2,2}\right)\right)\right.} \\
&\left.+\left(1-g_{1}^{*}\left(p_{1,1}, p_{1,2}\right)\right) p_{1,2} g_{2}^{*}\left(p_{2,1}, p_{2,2}\right)\right] \\
& d p_{2,2} d p_{2,1} d p_{1,2} d p_{1,1}
\end{aligned}
$$

In order to calculate this integration, we divide it into two cases: $p_{2,1} \geq p_{2,2}$ and $p_{2,1} \leq p_{2,2}$. For brevity, we only show how to calculate the case where $p_{2,1} \geq p_{2,2}$. The case where $p_{2,2}>p_{2,1}$ can be derived by using a similar method.

The integration is divided into the following 9 pieces when $p_{2,1}>p_{2,2}$.

- Case 1: When $p_{1,1} \in[0, \theta], p_{1,2} \in\left[0, p_{1,1}\right], p_{2,1} \in[0, \theta]$, $p_{2,2} \in\left[0, p_{2,1}\right]$, we have:

$$
\begin{gathered}
\frac{1}{4} \int_{0}^{\theta} \int_{0}^{p_{1,1}} \int_{0}^{\theta} \int_{0}^{p_{2,1}}\left(p_{1,1}+p_{1,2}\right) d p_{2,2} d p_{2,1} d p_{1,2} d p_{1,1} \\
=\frac{1}{16} \theta^{5}
\end{gathered}
$$

- Case 2: When $p_{1,1} \in[0, \theta], p_{1,2} \in\left[0, p_{1,1}\right], p_{2,1} \in(\theta, 1]$, $p_{2,2} \in\left[0, p_{2,1}-\theta\right)$, we have:

$$
\begin{array}{r}
\int_{0}^{\theta} \int_{0}^{p_{1,1}} \int_{\theta}^{1} \int_{0}^{p_{2,1}-\theta} \frac{1}{2} p_{1,2} d p_{2,2} d p_{2,1} d p_{1,2} d p_{1,1} \\
=\frac{1}{24} \theta^{3}-\frac{1}{12} \theta^{4}+\frac{1}{24} \theta^{5}
\end{array}
$$

- Case 3: When $p_{1,1} \in[0, \theta], p_{1,2} \in\left[0, p_{1,1}\right], p_{2,1} \in(\theta, 1]$, $p_{2,2} \in\left[p_{2,1}-\theta, p_{2,1}\right]$, we have:

$$
\begin{gathered}
\frac{1}{4} \int_{0}^{\theta} \int_{0}^{p_{1,1}} \int_{\theta}^{1} \int_{p_{2,1}-\theta}^{p_{2,1}}\left(p_{1,1}+p_{1,2}\right) d p_{2,2} d p_{2,1} d p_{1,2} d p_{1,1} \\
=\frac{1}{8} \theta^{4}-\frac{1}{8} \theta^{5}
\end{gathered}
$$

- Case 4: When $p_{1,1} \in(\theta, 1], p_{1,2} \in\left[0, p_{1,1}-\theta\right], p_{2,1} \in$ 
$[0, \theta], p_{2,2} \in\left[0, p_{2,1}\right]$, we have:

$$
\begin{gathered}
\int_{\theta}^{1} \int_{0}^{p_{1,1}-\theta} \int_{0}^{\theta} \int_{0}^{p_{2,1}} \frac{1}{2} p_{1,1} d p_{2,2} d p_{2,1} d p_{1,2} d p_{1,1} \\
=\frac{1}{12} \theta^{2}-\frac{1}{8} \theta^{3}+\frac{1}{24} \theta^{5}
\end{gathered}
$$

- Case 5: When $p_{1,1} \in(\theta, 1], p_{1,2} \in\left[0, p_{1,1}-\theta\right], p_{2,1} \in$ $(\theta, 1], p_{2,2} \in\left[0, p_{2,1}-\theta\right)$, we have:

$$
\int_{\theta}^{1} \int_{0}^{p_{1,1}-\theta} \int_{\theta}^{1} \int_{0}^{p_{2,1}-\theta} 0 d p_{2,2} d p_{2,1} d p_{1,2} d p_{1,1}=0
$$

- Case 6: When $p_{1,1} \in(\theta, 1], p_{1,2} \in\left[0, p_{1,1}-\theta\right], p_{2,1} \in$ $(\theta, 1], p_{2,2} \in\left[p_{2,1}-\theta, p_{2,1}\right]$, we have:

$$
\begin{aligned}
\int_{\theta}^{1} \int_{0}^{p_{1,1}-\theta} \int_{\theta}^{1} \int_{p_{2,1}-\theta}^{p_{2,1}} & \frac{1}{2} p_{1,1} d p_{2,2} d p_{2,1} d p_{1,2} d p_{1,1} \\
& =\frac{1}{6} \theta-\frac{5}{12} \theta^{2}+\frac{1}{4} \theta^{3} \\
& +\frac{1}{12} \theta^{4}-\frac{1}{12} \theta^{5}
\end{aligned}
$$

- Case 7: When $p_{1,1} \in(\theta, 1], p_{1,2} \in\left[p_{1,1}-\theta, p_{1,1}\right], p_{2,1} \in$ $[0, \theta], p_{2,2} \in\left[0, p_{2,1}\right]$, we have:

$$
\begin{aligned}
\frac{1}{4} \int_{\theta}^{1} \int_{p_{1,1}-\theta}^{p_{1,1}} \int_{0}^{\theta} \int_{0}^{p_{2,1}} & \left(p_{1,1}+p_{1,2}\right) d p_{2,2} d p_{2,1} d p_{1,2} d p_{1,1} \\
= & \frac{1}{8} \theta^{3}-\frac{1}{16} \theta^{4}-\frac{1}{16} \theta^{5}
\end{aligned}
$$

- Case 8: When $p_{1,1} \in(\theta, 1], p_{1,2} \in\left[p_{1,1}-\theta, p_{1,1}\right], p_{2,1} \in$ $(\theta, 1], p_{2,2} \in\left[0, p_{2,1}-\theta\right)$, we have:

$$
\begin{array}{r}
\int_{\theta}^{1} \int_{p_{1,1}-\theta}^{p_{1,1}} \int_{\theta}^{1} \int_{0}^{p_{2,1}-\theta} \\
\frac{1}{2} p_{1,2} d p_{2,2} d p_{2,1} d p_{1,2} d p_{1,1} \\
=\frac{1}{8} \theta-\frac{3}{8} \theta^{2}+\frac{3}{8} \theta^{3}-\frac{1}{8} \theta^{4}
\end{array}
$$

- Case 9: When $p_{1,1} \in(\theta, 1], p_{1,2} \in\left[p_{1,1}-\theta, p_{1,1}\right], p_{2,1} \in$ $(\theta, 1], p_{2,2} \in\left[p_{2,1}-\theta, p_{2,1}\right]$, we have:

$$
\begin{gathered}
\frac{1}{4} \int_{\theta}^{1} \int_{p_{1,1}-\theta}^{p_{1,1}} \int_{\theta}^{1} \int_{p_{2,1}-\theta}^{p_{2,1}} \\
\left(p_{1,1}+p_{1,2}\right) d p_{2,2} d p_{2,1} d p_{1,2} d p_{1,1} \\
=\frac{1}{4} \theta^{2}-\frac{3}{8} \theta^{3}+\frac{1}{8} \theta^{5}
\end{gathered}
$$

Summing equations from (5) through (13), we get the integration for the case $p_{2,1}>p_{2,2}$. When $p_{2,2}>p_{2,1}$, we get that the expected throughput in unit time ${ }^{2}$ is $\frac{1}{6}-\frac{7 \theta}{24}+\frac{\theta^{2}}{3}-$ $\frac{5 \theta^{3}}{24}+\frac{\theta^{4}}{16}$. Therefore, the expected throughput rate for user $P 1$ is:

$$
\mathbb{E}(R)=\frac{1}{6}-\frac{\theta^{2}}{8}+\frac{\theta^{3}}{12}
$$

In order to maximize $\mathbb{E}(R)$, we get $\theta=0$ and the maximal value of $\mathbb{E}(R)$ is 0.1667 . This is a somewhat surprising conclusion; it states that when there is no information about the other user, always pick the better of the two channels.

\footnotetext{
${ }^{2}$ We omit the calculation here for brevity.
}

Therefore, secondary user $P 1$ 's utility for conducting no negotiation is presented as follows:

$$
\widehat{U_{1}}=0.1667
$$

We now consider the other two cases: one round negotiation (i.e., exchanging partial information) and two-rounds negotiation (i.e., obtaining full information).

\subsubsection{Partial Information Exchange}

If the two users are involved in one-round negotiation, user $P 1$ will hear $p_{2,1}$ 's value or $p_{2,2}$ 's value with probability 0.5 each. Let $\mathbb{E}\left(R_{1}\right)$ and $\mathbb{E}\left(R_{2}\right)$ denote the expected throughput rate for user $P 1$ when hearing $p_{2,1}$ and $p_{2,2}$, respectively. Obviously, the expected throughput rate after one-round information exchange $\mathbb{E}(R)$ can be expressed as follows:

$$
\mathbb{E}(R)=\frac{1}{2} \mathbb{E}\left(R_{1}\right)+\frac{1}{2} \mathbb{E}\left(R_{1}\right)
$$

If user $P 1$ gets the information that user $P 2$ also prefers channel $C 1$ (i.e., $p_{2,1}$ is revealed to user $P 1$ ), we assume the optimal sensing decisions for both users follow the equation shown in equation (3).

We now focus on obtaining the expected utility for user $P 1$ hearing the value of $p_{2,1}$ after one round negotiation. Note that in this case, $P 1$ knows not only the value of $p_{2,1}$, but also is aware that $p_{2,1} \geq p_{2,2}$ according to the pre-defined negotiation rules. The expected utility of $P 1$ should be conditioned on these two facts.

Similar to the previous case where no negotiation happens, the expected throughput rate for user $P 1$ when getting $p_{2,1}$ can be expressed as follows:

$\mathbb{E}\left(R_{1}\right)=\int_{0}^{1} \int_{0}^{p_{1,1}} \int_{0}^{1} \int_{0}^{p_{2,1}} \Re() f\left(p_{2,1}\right) d p_{2,2} d p_{2,1} d p_{1,2} d p_{1,1}$

where $f\left(p_{2,1}\right)$ is the density of $p_{2,1}$ conditioning on $p_{2,1} \geq$ $p_{2,2}$ and $\Re()$ is defined below.

$$
\begin{aligned}
\Re() & =\left\{g_{1}^{*}\left(p_{1,1}, p_{1,2}\right) p_{1,1}\left(1-g_{2}^{*}\left(p_{2,1}, p_{2,2}\right)\right)\right. \\
& \left.+\left(1-g_{1}^{*}\left(p_{1,1}, p_{1,2}\right)\right) p_{2,2} g_{2}^{*}\left(p_{2,1}, p_{2,2}\right)\right\} f\left(p_{2,2}\right)
\end{aligned}
$$

In equation 18, $f\left(p_{2,2}\right)$ denotes the conditional density of $p_{2,2}$ when given $p_{2,1} \geq p_{2,2}$. That is, $f\left(p_{2,2}\right)=\frac{1}{p_{2,1}}$. In the calculation below, we consider two independent cases where $p_{2,1} \leq \theta$ and $p_{2,1}>\theta$. In order to make the description clear, we develop some notation:

$$
\begin{aligned}
\Gamma & =\int_{0}^{1} \int_{0}^{p_{2,1}} \Re() f\left(p_{2,1}\right) d p_{2,2} d p_{2,1} \\
\Gamma_{l} & =\int_{0}^{\theta} \int_{0}^{p_{2,1}} \Re() f\left(p_{2,1}\right) d p_{2,2} d p_{2,1} \\
\Gamma_{h} & =\int_{\theta}^{1} \int_{0}^{p_{2,1}} \Re() f\left(p_{2,1}\right) d p_{2,2} d p_{2,1}
\end{aligned}
$$

It is straightforward that $\Gamma=\Gamma_{l}+\Gamma_{h}$. We first calculate $\Gamma_{l}$ and then consider $\Gamma_{h}$. 
We have $g_{2}^{*}\left(p_{2,1}, p_{2,2}\right)=0.5$ when $p_{2,1} \leq \theta$. Substitute $g_{2}^{*}($.$) and f\left(p_{2,2}\right)$ to equation 20 , we have:

$$
\begin{aligned}
\Gamma_{l} & =\frac{1}{2} \int_{0}^{\theta} \int_{0}^{p_{2,1}}\left\{g_{1}^{*}\left(p_{1,1}, p_{1,2}\right) p_{1,1}\right. \\
& \left.+\left(1-g_{1}^{*}\left(p_{1,1}, p_{1,2}\right)\right) p_{1,2}\right\} f\left(p_{2,2}\right) d p_{2,2} d p_{2,1} \\
& =\frac{1}{2} \int_{0}^{\theta}\left[g_{1}^{*}\left(p_{1,1}, p_{1,2}\right) p_{1,1}\right. \\
& \left.+\left(1-g_{1}^{*}\left(p_{1,1}, p_{1,2}\right)\right) p_{1,2}\right] f\left(p_{2,1}\right) d p_{2,1}
\end{aligned}
$$

For the case where $p_{2,1}>\theta$, we consider the following two cases in order to decide the value of $g_{2}^{*}($.$) .$

$$
g_{2}^{*}\left(p_{2,1}, p_{2,2}\right)=\left\{\begin{aligned}
0.5 & \left.\left(p_{2,2} \geq p_{2,1}-\theta\right)\right) \\
1 & \left(p_{2,2}<p_{2,1}-\theta\right)
\end{aligned}\right.
$$

By replacing the corresponding $g_{2}^{*}($.$) value in equation 21$, we get the following expression. Note that for brevity, in this equation, we use $g_{i}^{*}($.$) to denote g_{i}^{*}\left(p_{i, 1}, p_{i, 2}\right)$.

$$
\begin{aligned}
\Gamma_{h} & =\int_{\theta}^{1} \int_{0}^{p_{2,1}-\theta}\left[g_{1}^{*}(.) p_{1,1}\left(1-g_{2}^{*}(.)\right)\right. \\
& +\left(1-\left(g_{1}^{*}(.) p_{2,2} g_{2}^{*}(.)\right] f\left(p_{2,2}\right) f\left(p_{2,1}\right) d p_{2,2} d p_{2,1}\right. \\
& +\int_{\theta}^{1} \int_{p_{2,1}-\theta}^{p_{2,1}}\left[g_{1}^{*}(.) p_{1,1}\left(1-g_{2}^{*}(.)\right)\right. \\
& \left.+\left(1-g_{1}^{*}(.)\right) p_{2,2} g_{2}^{*}(.)\right] f\left(p_{2,2}\right) f\left(p_{2,1}\right) d p_{2,2} d p_{2,1} \\
& =\int_{\theta}^{1} \int_{0}^{p_{2,1}-\theta}\left(1-g_{1}^{*}(.)\right) p_{1,2} f\left(p_{2,2}\right) f\left(p_{2,1}\right) d p_{2,2} d p_{2,1} \\
& +\frac{1}{2} \int_{\theta}^{1} \int_{p_{2,1}-\theta}^{p_{2,1}}\left[g_{1}^{*}(.) p_{1,1}+(1\right. \\
& \left.\left.-g_{1}^{*}(.)\right) p_{1,2}\right] f\left(p_{2,2}\right) f\left(p_{2,1}\right) d p_{2,2} d p_{2,1} \\
& =\int_{\theta}^{1}\left\{\left[\left(1-g_{1}^{*}(.)\right) p_{1,2}\right] \frac{p_{2,1}-\theta}{p_{2,1}}\right. \\
& \left.+\frac{1}{2}\left[g_{1}^{*}(.) p_{1,1}+\left(1-g_{1}^{*}(.)\right) p_{1,2}\right] \frac{\theta}{p_{2,1}}\right\} f\left(p_{2,1}\right) d p_{2,1}
\end{aligned}
$$

Now we will focus on solving $f\left(p_{2,1}\right)$. Note that $p_{2,1}$ is not uniformly distributed when considering that $p_{2,1}$ is the larger number of the two random variables $p_{2,1}$ and $p_{2,2}$. In fact, the probability that user $P 1$ sees $p_{2,1} \leq z(z \in[0,1])$ when given that $p_{2,1}$ is no less than $p_{2,2}$ (i.e., $p_{2,1} \geq p_{2,2}$ ) can be computed as follows:

$$
\begin{aligned}
\operatorname{Pr}\left(p_{2,1} \leq z \mid p_{2,1} \geq p_{2,2}\right) & =\frac{\operatorname{Pr}\left(p_{2,1} \leq z, p_{2,1} \geq p_{2,2}\right)}{\operatorname{Pr}\left(p_{2,1} \geq p_{2,2}\right)} \\
& =\frac{\int_{0}^{z} \int_{0}^{p_{2,1}} d p_{2,2} d p_{2,1}}{\operatorname{Pr}\left(p_{2,1} \geq p_{2,2}\right)}=\frac{z^{2} / 2}{1 / 2} \\
& =z^{2}
\end{aligned}
$$

Therefore, $f\left(p_{2,1}\right)=2 p_{2,1}$. Thus,

$$
\begin{aligned}
\Gamma & =\Gamma_{l}+\Gamma_{h} \\
& =\int_{0}^{\theta}\left[g_{1}^{*}\left(p_{1,1}, p_{1,2}\right) p_{1,1}+\left(1-g_{1}^{*}\left(p_{1,1}, p_{1,2}\right)\right) p_{1,2}\right] p_{2,1} d p_{2,1} \\
& +\int_{\theta}^{1}\left\{2\left[\left(1-g_{1}^{*}\left(p_{1,1}, p_{1,2}\right)\right) p_{1,2}\right] \frac{p_{2,1}-\theta}{p_{2,1}}\right. \\
& \left.+\left[g_{1}^{*}\left(p_{1,1}, p_{1,2}\right) p_{1,1}+\left(1-g_{1}^{*}\left(p_{1,1}, p_{1,2}\right)\right) p_{1,2}\right] \frac{\theta}{p_{2,1}}\right\} p_{2,1} d p_{2,1}
\end{aligned}
$$

User $P 1$ 's expected throughput rate after one round negotiation on hearing $p_{2,1}$ can be expressed as:

$$
\mathbb{E}\left(R_{1}\right)=\int_{0}^{1} \int_{0}^{p_{1,1}} \Gamma d p_{1,2} d p_{1,1}
$$

Again, this integration can be divided to the following three different cases according to the value of $g_{1}^{*}\left(p_{1,1}, p_{1,2}\right)$ : 1) if $\left.p_{1,1} \leq \theta, g_{1}^{*}\left(p_{1,1}, p_{1,2}\right)=0.5 ; 2\right)$ if $p_{1,1}>\theta$ and $p_{1,2} \geq$ $\left.p_{1,1}-\theta, g_{1}^{*}\left(p_{1,1}, p_{1,2}\right)=0.5 ; 3\right)$ if $p_{1,1}>\theta$ and $0 \leq p_{1,2}<$ $p_{1,1}-\theta, g_{1}^{*}\left(p_{1,1}, p_{1,2}\right)=1$.

After solving the integration, we get:

$$
\mathbb{E}\left(R_{1}\right)=\frac{7 \theta^{3}}{12}-\frac{\theta^{4}}{8}-\frac{11 \theta^{2}}{12}+\frac{7 \theta}{12}
$$

Now we consider the other case on conducting one round negotiation. If user $P 1$ gets user $P 2$ 's channel $C 2$ valuation $p_{2,2}$, the optimal sensing policy is decided immediately (user $P 1$ will sense channel $C 1$ and user $P 2$ will sense channel $C 2$ ). In this case, user $P 1$ 's expected throughput rate equals the expected value of $p_{1,1}$. Note that in our assumption, $p_{1,1} \geq p_{1,2} \cdot p_{1,1}$ is not uniformly distributed any more. The expected value of random variable $p_{1,1}$ equals the expected value for $\max \left(p_{1,1}, p_{1,2}\right)$. Hence, we have

$$
\mathbb{E}\left(p_{1,1}\right)=\frac{2}{3}
$$

According to equation 16, user $P 1$ 's expected throughput in unit time after one round negotiation is:

$$
\begin{aligned}
\mathbb{E}(R) & =\frac{1}{2} \mathbb{E}\left(R_{1}\right)+\frac{1}{2} \mathbb{E}\left(R_{2}\right) \\
& =\frac{7 \theta^{3}}{24}-\frac{\theta^{4}}{16}-\frac{11 \theta^{2}}{24}+\frac{7 \theta}{24}+\frac{1}{3}
\end{aligned}
$$

The maximal value is 0.3982 and it is obtained at $\theta=0.62$. Therefore, when considering exchange information in oneround negotiation, the expected utility for user $P 1$ can be expressed as follows:

$$
\widehat{U_{1}}=0.3982(1-\beta)
$$

\subsubsection{Full Information Exchange}

Finally, we consider the case where both users gains full information about each other after two rounds of negotiation. Similar to the previous two cases, we will first calculate the expected throughput rate as if the channel valuations were given. Then we average on all the possible valuations by integration.

When global information is available about all user valuations of all channels, the problem of obtaining the channel allocation that maximizes the sum rate for the two users 
is essentially a maximum weight bipartite matching problem [6]. In general, this problem can be solved efficiently in polynomial time using the Hungarian algorithm [13]. For the special case of two users and two channels that we have here, the optimal solution can be described explicitly in terms of the valuations of each users as follows. There are three possible outcomes for user $P 1$ : a) $P 1$ senses $C 1$ if $p_{1,1}+p_{2,2}>$ $p_{1,2}+p_{2,1}$; b) $P 1$ senses $C 2$ if $p_{1,1}+p_{2,2}<p_{1,2}+p_{2,1}$; c) $P 1$ randomly chooses either $C 1$ or $C 2$ with probability $\frac{1}{2}$ if $p_{1,1}+p_{2,2}=p_{1,2}+p_{2,1}$. The solution for user $P 2$ is symmetric.

As we mentioned before, in our average analysis, although we are maximizing user $P 1$ 's expected throughput rate in previous two cases, user $P 2$ 's expected throughput rate is also optimized due to symmetry. Hence, the system throughput rate is maximized in previous two cases as well, making them all comparable.

We first suppose that user $P 1$ already knows the value of $p_{1,1}-p_{1,2}$, denoted as $p_{\text {diff }}$. Let $\mathbb{E}(R \mid V)$ denote the expected throughput in unit time for user $P 1$ when user $P 1$ already knows his/her own channel valuations. We have:

$$
\begin{aligned}
\mathbb{E}(R \mid V) & =p_{1,1} \operatorname{Pr}\left(p_{2,1}-p_{2,2}<p_{\text {diff }}\right) \\
& +p_{1,2} \operatorname{Pr}\left(p_{2,1}-p_{2,2}>p_{\text {diff }}\right) \\
& +\frac{p_{1,1}+p_{1,2}}{2} \operatorname{Pr}\left(p_{2,1}-p_{2,2}=p_{\text {diff }}\right)
\end{aligned}
$$

Considering that $p_{1,1}, p_{1,2}, p_{2,1}, p_{2,2}$ are uniformly distributed in the interval $[0,1]$, we have

$$
\begin{gathered}
\operatorname{Pr}\left(p_{2,1}-p_{2,2}<p_{\text {diff }}\right)=1-\frac{\left(1-p_{\text {diff }}\right)^{2}}{2} \\
\operatorname{Pr}\left(p_{2,1}-p_{2,2}>p_{\text {diff }}\right)=\frac{\left(1-p_{\text {diff }}\right)^{2}}{2}
\end{gathered}
$$

and

$$
\operatorname{Pr}\left(p_{2,1}-p_{2,2}=p_{\text {diff }}\right)=0
$$

. Substituting these values to equation (30), we have:

$$
\mathbb{E}(R \mid V)=p_{1,1}-\frac{p_{\text {diff }}\left(1-p_{\text {diff }}\right)^{2}}{2}
$$

According to linearity of expectation, in order to get $\mathbb{E}(R)$ for user $P 1$ from this equation, we need to calculate the expected value of $p_{1,1}$ and $p_{\text {diff }}\left(1-p_{\text {diff }}\right)^{2}$. The expected value of $p_{1,1}$ has already been calculated in equation 27 . Now we focus on computing the expectation of $p_{\text {diff }}\left(1-p_{\text {diff } f}\right)^{2}$ on the assumption that $p_{1,1} \geq p_{1,2}$.

Expanding $p_{\text {diff } f}\left(1-p_{\text {diff }}\right)^{2}$, we have:

$$
\begin{aligned}
p_{\text {diff } f}\left(1-p_{\text {diff }}\right)^{2} & =\frac{\left(p_{1,1}-p_{1,2}\right)\left[1-\left(p_{1,1}-p_{1,2}\right)\right]^{2}}{2} \\
& =\frac{1}{2}\left(p_{1,1}-p_{1,2}\right)-\left(p_{1,1}-p_{1,2}\right)^{2} \\
& +\frac{1}{2}\left(p_{1,1}-p_{1,2}\right)^{3}
\end{aligned}
$$

Now we need to calculate the conditional expectation for $\left(p_{1,1}-p_{1,2}\right)^{n}(n=1,2,3)$ when given that $p_{1,1}>p_{1,2}$.

$$
\begin{aligned}
& \mathbb{E}\left(\left(p_{1,1}-p_{1,2}\right)^{n} \mid p_{1,1} \geq p_{1,2}\right) \\
= & \mathbb{E}\left(\left(\mathbb{E}\left(\left(p_{1,1}-p_{1,2}\right)^{n} \mid p_{1,1}, p_{1,1} \geq p_{1,2}\right)\right) \mid p_{1,1} \geq p_{1,2}\right)
\end{aligned}
$$

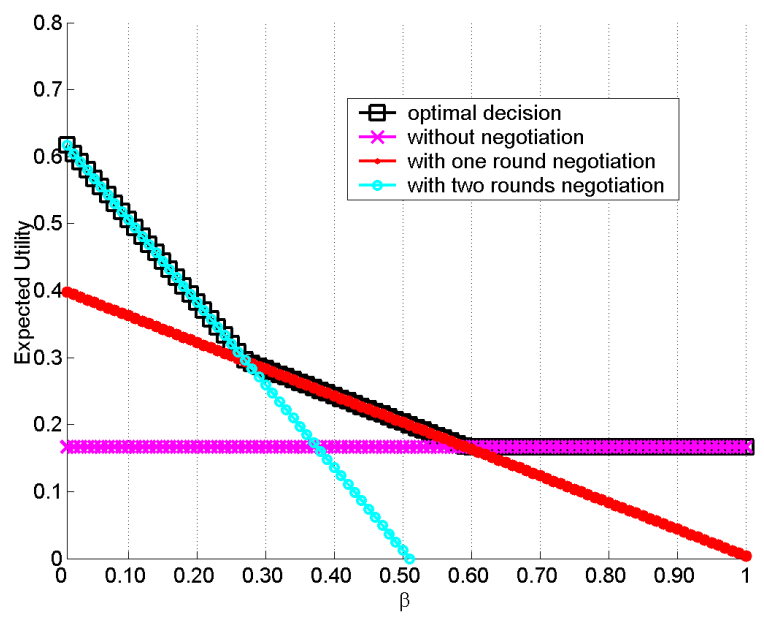

Figure 2: Illustration of user $P 1$ 's optimal decision on number of negotiation rounds

By applying the above fact, we can obtain:

$$
\begin{aligned}
& \mathbb{E}\left(\left(p_{1,1}-p_{1,2}\right)^{n} \mid p_{1,1}, p_{1,1} \geq p_{1,2}\right) \\
= & \int_{0}^{p_{1,1}}\left(p_{1,1}-p_{1,2}\right)^{n} \frac{1}{p_{1,1}} d p_{1,2} \\
= & -\left.\frac{1}{p_{1,1}} \cdot \frac{\left(p_{1,1}-p_{1,2}\right)^{n+1}}{n+1}\right|_{0} ^{p_{1,1}} \\
= & \frac{p_{1,1}^{n}}{n+1}
\end{aligned}
$$

Thus,

$$
\begin{aligned}
\mathbb{E}\left(\left(p_{1,1}-p_{1,2}\right)^{n} \mid p_{1,1} \geq p_{1,2}\right) & =\int_{0}^{1} \frac{p_{1,1}^{n}}{n+1} 2 p_{1,1} d p_{1,1} \\
& =\frac{2}{(n+1)(n+2)}
\end{aligned}
$$

Substituting the corresponding value in equation 31 , we get the value of $\mathbb{E}(R)$ for user $P 1$ as follows:

$$
\mathbb{E}(R)=\frac{2}{3}-\left(\frac{1}{2} \cdot \frac{1}{3}-\frac{1}{6}+\frac{1}{2} \cdot \frac{1}{10}\right)=\frac{37}{60}
$$

The expected utility for user $P 1$ after full information exchanged can be represented by the following equation:

$$
\widehat{U_{1}}=\frac{37}{60}(1-2 \beta)
$$

\subsection{Optimal Information Exchange}

In previous section, we have obtained the optimal threshold value $\theta$ and the expression of user $P 1$ 's expected utility functions for the three cases: without negotiation, with one round negotiation, and with two rounds negotiation. Users decide the number of negotiation rounds a priori according to the optimal threshold value such that their expected utility $\widehat{U_{i}}$ is maximized. Figure 2 illustrates user $P 1$ 's optimal negotiation round decision according to the negotiation cost (i.e., $\beta$ value).

The implication of figure 2 is straightforward. When $\beta$ is small (i.e., each round of negotiation occupies a small potion of the usable given time period), the user prefers 
to get perfect information about the system before making channel sensing decisions. With the increase of $\beta$, getting partial information of the other user becomes the optimal strategy in terms of total expected throughput in the given time interval. Only when $\beta$ is large enough (around 0.68 in the plot), the user's best strategy is to begin transmit without any information exchanging. Notice that $\beta=0.68$ implies a large overhead on negotiation cost. In most cases, the secondary users prefer to gather some information from the opponent to improve the expected utility.

\section{CONCLUSION}

In this paper, we have investigated the problem of how secondary users in cognitive radio networks should coordinate with each other on channel sensing and access. In this scenario, each secondary user has his/her own valuation on channel conditions. The channel valuations are independent and uniformly randomly distributed between 0 and 1 .

At the beginning of a given time period, the secondary users need to decide intelligently on the number of negotiation round to maximize their expected throughput in this given time interval. There is a tradeoff between information gathering and channel using. On the one hand, exchanging channel valuation information among secondary users might help improving the user's throughput in the given period by reducing collisions among secondary users. On the other hand, information exchange takes time.

We have proposed a threshold-based channel sensing policy based on an observation of our previous work to handle the ambiguous case where the channel sensing policy is not implicitly determined. We have obtained the optimal threshold for both users to maximize their expected throughput. Finally, we have discussed how users should choose optimal negotiation round a priori according to different negotiation costs.

There are many open problems and directions to consider in future work. One is to consider alternatives to the threshold policy we have used in this work, or to prove that the threshold policy is indeed the best possible in some sense. Another possible research direction is to extend the current study to scenarios with more than 2 users and more than 2 channels. Yet another direction of interest to us is to consider more dynamic settings, where the users decide whether to terminate or continue the negotiation process at the end of each negotiation round based on the information gathered up to that point.

\section{REFERENCES}

[1] Q. Zhao and B.M. Sadler, "A Survey of Dynamic Spectrum Access" in IEEE Signal Processing Magazine, vol. 24, no. 3, pp. 79-89, May, 2007.

[2] Q. Zhao, L. Tong, A. Swami, and Y. Chen, "Decentralized Cognitive MAC for Opportunistic Spectrum Access in Ad Hoc Networks: A POMDP Framework," IEEE Journal on Selected Areas in Communications (JSAC): Special Issue on Adaptive, Spectrum Agile and Cognitive Wireles Networks, vol. 25, no. 3, pp. 589-600, April, 2007.

[3] L. Cao, H. Zheng, "Distributed spectrum allocation via local bargaining," IEEE SECON 2005. Second Annual IEEE Communications Society Conference on Sensor and Ad Hoc Communications and Networks, 2005. pp. 475-486, 26-29 Sept., 2005.
[4] N. Chang and M. Liu, "Optimal channel probing and transmission scheduling for opportunistic spectrum access", in Proc. ACM International Conference on Mobile Computing and Networking (MobiCom), September 2007, Montreal, Canada.

[5] F. Fu, M. van der Schaar, "Learning to Compete for Resources in Wireless Stochastic Games," IEEE Trans. Veh. Tech., to appear.

[6] H. Liu, B. Krishnamachari, "Randomized Strategies for Multi-User Multi-Channel Opportunity Sensing," Cognitive Radio Networks Workshop, IEEE CCNC 2008, Las Vegas, Nevada.

[7] H. Liu, B. Krishnamachari, Q. Zhao, "Cooperation and Learning in Multiuser Opportunistic Spectrum Access," Cognet Workshop. IEEE ICC 2008, Beijing, China.

[8] Q. Zhao, B. Krishnamachari, "Structure and Optimality of Myopic Sensing for Opportunistic Spectrum Access", Cognet Workshop, IEEE ICC 2007.

[9] Q. Zhao, B. Krishnamachari, and K. Liu "On Myopic Sensing for Multi-Channel Opportunistic Access: Structure, Optimality, and Performance" IEEE Transactions on Wireless Communications, to appear, 2008.

[10] T. Javidi, B. Krishnamachari, Q. Zhao, and M. Liu "Optimality of Myopic Sensing in Multi-Channel Opportunistic Access", Proc. of IEEE International Conference on Communications (ICC), May, 2008.

[11] S.H. Ahmad, M. Liu, T. Javidi, Q. Zhao and B. Krishnamachari "Optimality of Myopic Sensing in Multi-Channel Opportunistic Access" submitted to IEEE Transactions on Information Theory, 2008.

[12] K. Liu, Q. Zhao, and Y. Chen "Distributed Sensing and Access in Cognitive Radio Networks" 10th International Symposium on Spread Spectrum Techniques and Applications (ISSSTA), August, 2008

[13] H. W. Kuhn, "The Hungarian Method for the assignment problem", Naval Research Logistics Quarterly, 2:83-97, 1955.

[14] H. Liu, B. Krishnamachari, Q. Zhao, "Negotiating Multichannel Sensing and Access In Cognitive Radio Wireless Networks," USC CENG technical report CENG-2008-14, October 2008.

[15] A. B. MacKenzie and S. B. Wicker, "Selfish Users in Aloha: A Game-Theoretic Approach, ک̌ IEEE Vehicular Technology Conference, October 7-11, 2001.

[16] M. M. Halldorsson, J. Y. Halpern, L. Li, V.S. Mirrokni, "On spectrum sharing games," In Proceedings of the Twenty-Third Annual ACM Symposium on Principles of Distributed Computing (PODC), 2004.

[17] J. Konorski, "A Game-Theoretic Study of CSMA/CA Under a Backoff Attack," IEEE/ACM Transactions on Networking, December 2006.

[18] A.R. Cassandra, POMDP solver in C, Version 4.0, http://pomdp.org/pomdp/code/index.shtml. 